\title{
Everolimus, Cyclosporine, and Thrombotic Microangiopathy: Clinical Role and Preventive Tools in Renal Transplantation
}

\author{
F. Nava*, G. Cappelli, G. Mori, M. Granito, G. Magnoni, C. Botta, A. Solazzo, F. Fontana, A. Baisi, \\ and D. Bonucchi \\ Nephrology Dialysis and Kidney Transplantation, AOU Policlinico, Modena, Italy
}

\begin{abstract}
Introduction. Thrombotic microangiopathy (TMA) is characterized by endothelial cell injury and formation of fibrin thrombi within capillary and arterioles. In renal allograft recipients, TMA mainly presents as hemolytic uremic syndrome. Its occurrence is rare, and diagnosis requires a high degree of suspicion. Drug toxicity, in particular from calcineurin inhibitors (CNIs) and mTOR inhibitors (mTORi), is the most common cause posttransplant and has recently been emphasized in the setting of lung transplantation.

Objective. The goal of this study was to investigate the role of mTORi as an added risk factor in the development of TMA to propose strategies for modulation of immunosuppressive (IS) therapy.

Patients and Methods. From a database of 496 renal graft recipients, we analyzed 350 renal graft biopsy specimens gathered at our center from 1998 to 2012. In patients undergoing combined therapy with mTORi and CNI, we compared drugs levels in TMA-affected and TMA-free groups, using mTORi and CNI TLC and the summation of [everolimus TLC + (cyclosporine $\mathrm{C} 2 / 100)](\Sigma)$ as a surrogate marker of combined exposition to 2 drugs. Receiver-operating characteristic analysis of association of EVL TLC $+(\mathrm{C} 2 / 100)$ was performed for patients exposed to mTORi.

Results. Histologic features of TMA were found in 36 patients (prevalence of $7.3 \%$ ). The caseload was divided into 2 groups: not drug-related TMA $(n=19)$ and drug-related TMA $(n=17)$. Despite the prevalence of TMA in patients exposed to mTORi being greater $(8$ of 153; prevalence, $5.3 \%$ ) compared with therapies without mTORi (9 of 324; prevalence, $2.8 \%$ ), statistical difference was not reached. Patients treated with mTORi who developed de novo drug-related TMA had higher blood levels of IS drugs compared with those who did not develop TMA. Receiver-operating characteristic analysis found a significant threshold of $12.5 \mathrm{ng} / \mathrm{mL}$ (area under the curve, $0.803 ; P=.006$ ).

Conclusions. Results confirm the pivotal role of IS drugs in the onset of de novo TMA. On the basis of literature, we could speculate a sequence of endothelial damage by CNI, on which everolimus fits hindering the repair of endothelial injury. Therefore, high blood levels of CNI and mTORi seem to predispose patients to posttransplant TMA. Combined monitoring of these 2 drugs might be used to prevent the complication. $\Sigma$ [everolimus TLC + (cyclosporine $\mathrm{C} 2 / 100)]>12.5 \mathrm{ng} / \mathrm{mL}$ should be avoided as a surrogate risk factor for adverse effects.
\end{abstract}

T HROMBOTIC MICROANGIOPATHY (TMA) is a pathologic condition characterized by the formation of fibrin thrombi and platelet aggregations within capillary and arterioles leading to endothelial cell injury and small vessel occlusion. This causes thrombocytopenia, hemolytic anemia,

(c) 2014 by Elsevier Inc. All rights reserved.

360 Park Avenue South, New York, NY 10010-1710
*Address correspondence to Dr. Fabio Nava (MD, resident) Nephrology Dialysis and Kidney Transplantation, AOU Policlinico, Modena, Italy. E-mail: fabio.nava24k@gmail.com

$0041-1345 / 14$

http://dx.doi.org/10.1016/j.transproceed.2014.07.062 
purpura, and renal failure. TMA mainly presents as hemolytic uremic syndrome (HUS) and thrombotic thrombocytopenic purpura; although HUS is distinguished by the presence of more severe and refractory renal involvement, thrombotic thrombocytopenic purpura has a typical neurologic involvement and depends on ADAMTS13 dysfunction.

In renal allograft recipients, HUS is rare, with an incidence ranging between $0.8 \%$ and $14 \%$ [1]. When unrecognized and not treated early, it can lead to graft loss and, in severe cases, death. Toxicity by calcineurin inhibitors (CNIs) and mTOR inhibitors (mTORi), viral infection, ischemiareperfusion injury, antibody-mediated rejection (AMR), and presence of antiphospholipid antibodies [2] are the most frequent etiologies. Drug toxicity, in particular from CNIs, is the most common cause of posttransplant HUS; TMA has been a well-known adverse effect of cyclosporine (CsA) since its first use in transplantation [3]. Systemic signs of CsA-related TMA are uncommon, underscoring the importance of a graft biopsy for diagnosis. Conversely, mTORi sirolimus (SIR) and everolimus (EVE) inhibit glomerular endothelial cell proliferation and vascular endothelial growth factor activity in experimental TMA $[4,5]$. This could delay the repair of endothelial injury caused by CNI cytotoxicity as a first step in posttransplant TMA pathogenesis. A recent study on lung transplantation found that EVE and CsA association induced a high risk of TMA/HUS (related to the drugs' plasma levels) [6].

We retrospectively analyzed our transplant caseload with 3 main objectives: (1) to estimate the prevalence of biopsyproven TMA in our renal transplant patients; (2) to evaluate the role of immunosuppressive (IS) combinations and their levels in the onset of TMA, with particular focus on mTORi; and (3) to determine a surrogate indicator of toxicity by IS combinations (CsA minimization and EVE).

\section{MATERIALS AND METHODS}

We analyzed 350 renal graft biopsy specimens gathered from 1998 to 2012 in 496 renal graft recipients (excluding kidney/pancreas, kidney/ liver allografts, and primary nonfunction). Patients provided written informed consent before graft biopsy; the biopsy was indicated on clinical grounds (worsening renal function and/or proteinuria, signs of hemolitic anemia such as lactate dehydrogenase elevation, without other explanation). No protocol biopsies were performed.

In each patient with biopsy-proven TMA, we evaluated therapy at the time of biopsy and time from transplantation at the onset, interventions, and outcome expressed as graft loss. The cases were divided into 2 groups: not drug-related TMA and drug-related TMA. In the first group, TMA was related to various clinical pathologic conditions; for example, AMR, viral infections, systemic lupus erythematosus, and recurrence of atypical HUS. In the second group, TMA was linked exclusively to IS drugs.

Mean IS drug levels were measured in patients exposed to mTORi comparing TMA and TMA-free groups. To ensure the evaluation of a stabilized IS therapy, for the first group we considered the 3 months before biopsy, taking into account peak values before the procedure.

For the latter (TMA-free as control subjects), we analyzed TLCs for 6 months, after a minimum of 6 months of stabilized mTORi therapy. For statistical comparisons between patients with TMA who were exposed or not exposed to mTORi, and between drugrelated and not drug-related TMA groups, a $\chi^{2}$ analysis with Yates' correction was used. Drug blood levels were compared by using the Student unpaired $t$ test or nonparametric Mann-Whitney $U$ test where appropriate. SPSS (IBM SPSS Statistics 21.0, IBM Corporation, Armonk, NY, United States) was used for statistical analysis, and $P<.05$ was considered statistically significant. Data are presented as medians with ranges. Receiver-operating characteristic analysis of the association of EVL TLC $+(\mathrm{C} 2 / 100)$ was performed for patients exposed to mTORi.

\section{RESULTS}

Thirty-six cases of TMA were found in 350 renal biopsy specimens in a population of 496 patients receiving a renal allograft. Prevalence was $7.3 \%$ (36 of 496). TMA that was not drug related $(\mathrm{n}=19)$ included AMR $(\mathrm{n}=11)$, viral infection (cytomegalovirus, human immunodeficiency virus) $(\mathrm{n}=5)$, vasculitis $(\mathrm{n}=1)$, systemic lupus erythematosus $(\mathrm{n}=1)$, and recurrent atypical HUS $(\mathrm{n}=1)$; drug-related TMA was found in $3.4 \%$ of patients $(n=17)$. Statistical analysis found no significant differences between the 2 groups (drug related and not drug related TMA), except for distribution of histopathologic features of rejection, resulting prevalent in not drug related group (Tables 1 and 2).

The present study focused on the 17 patients of the drugrelated TMA group to investigate the specific role of IS drugs. Drug-related TMA group prevalence was 3.4\% (17 of 496). Patients with drug-related TMA did not differ significantly with respect to age ( $52 \pm 12$ years) and sex ( 9 male subjects, 8 female subjects). The allografts were single grafts from a cadaveric donor in 14 of 17 cases. In 2 cases, it was a double renal transplant; only 1 was from a living related donor. The mean time between transplantation and onset of de novo TMA was 276 days (range, 9 days to 4 years).

Eight cases of TMA were found in the mTORi-treated group $(\mathrm{n}=153$; prevalence, $5.3 \%)$; 6 were associated with CsA-EVE and 2 with FK-EVE. Nine cases of TMA were observed in patients not receiving mTORi $(\mathrm{n}=324)$, with a prevalence of $2.8 \%$; of these, 8 were treated with FK506 and 1 with CsA. A $\chi^{2}$ analysis with Yates' correction yielded no significant difference in the prevalence of TMA between the 2 groups $(P=.28)$. There were no cases of TMA in our patients treated with SIR.

Patients treated with mTORi who developed de novo drugrelated TMA had higher blood levels of IS drugs compared with those who did not develop TMA. In particular, serum CsA TLC (C0) was $134.8 \pm 108.1 \mathrm{ng} / \mathrm{mL}$ in patients with TMA (6 cases) and $53.6 \pm 23.6 \mathrm{ng} / \mathrm{mL}$ in patients without TMA treated with CsA $(P<.05)$. C2 levels were statistically nonsignificant, despite higher values in cases with TMA $(588.8 \pm 433.2 \mathrm{ng} / \mathrm{mL}$ vs $317.0 \pm 95.5 \mathrm{ng} / \mathrm{mL} ; P=.095)$. EVE TLC mean values, when used in association with CsA, were $9.9 \pm 3.9 \mathrm{ng} / \mathrm{mL}$ in patients with TMA and $7.5 \pm 2.2 \mathrm{ng} / \mathrm{mL}$ in patients without TMA $(P=.0381)$. Noteworthy was the difference $(P=.034)$ between the summation of concentrations of the 2 drugs: $\Sigma$ $[\mathrm{EVE}$ TLC $+(\mathrm{C} 2 / 100)]: 15.2 \pm 6.3 \mathrm{ng} / \mathrm{mL}$ in patients with 
Table 1. Renal Graft Recipients With Drug-Related TMA

\begin{tabular}{|c|c|c|c|c|c|c|c|c|c|c|c|c|c|}
\hline Patient & Sex & Age $(y)$ & $\begin{array}{l}\text { Native Renal } \\
\text { Disease }\end{array}$ & Allograft & $\begin{array}{c}\text { TMA Onset } \\
\text { Posttransplant (d) }\end{array}$ & Prebiopsy Therapy & CMV & HIV & $\mathrm{HR}$ & DSA & C4d & LDH & Blood Smear \\
\hline 1 & $\mathrm{M}$ & 35 & Alport & Living & 128 & FK + EVE & $\mathrm{Neg}$ & $\mathrm{Neg}$ & Neg & - & - & $=$ & - \\
\hline 2 & $\mathrm{~F}$ & 45 & Pyeloneph & Single & 253 & CsA + EVE & Neg & $\mathrm{Neg}$ & $\mathrm{Neg}$ & $\mathrm{Neg}$ & $\mathrm{Neg}$ & $\uparrow$ & $\mathrm{Neg}$ \\
\hline 3 & $\mathrm{M}$ & 39 & VUR & Single & 1494 & CsA + EVE & $\mathrm{Neg}$ & $\mathrm{Neg}$ & Neg & $\mathrm{Neg}$ & Neg & $=$ & Neg \\
\hline 4 & $M$ & 44 & $\operatorname{IgAN}$ & Single & 138 & $\mathrm{CsA}+\mathrm{EVE}$ & $\mathrm{Neg}$ & Neg & $\mathrm{Neg}$ & Neg & Pos & $\uparrow$ & $\mathrm{Neg}$ \\
\hline 5 & $\mathrm{~F}$ & 66 & Lithiasis & Double & 343 & $\mathrm{CsA}+\mathrm{EVE}$ & $\mathrm{Neg}$ & Neg & $\mathrm{Neg}$ & I & Pos & $\uparrow$ & Neg \\
\hline 6 & $\mathrm{~F}$ & 60 & Interstitial & Single & 109 & $\mathrm{FK}+\mathrm{EVE}$ & $\mathrm{Neg}$ & Neg & Neg & Neg & Neg & $\uparrow$ & Neg \\
\hline 7 & $\mathrm{~F}$ & 61 & DN & Single & 33 & $\mathrm{CsA}+\mathrm{EVE}$ & Neg & Neg & $\mathrm{Neg}$ & - & Neg & $\uparrow$ & Hemolysis \\
\hline 8 & $M$ & 64 & $\mathrm{DN}$ & Single & 254 & $\mathrm{Cs} A+\mathrm{EVE}$ & Neg & Neg & $\mathrm{Neg}$ & II & - & $\uparrow$ & - \\
\hline 9 & $M$ & 54 & Extracapillary GN & Single & 12 & FK & $\mathrm{Neg}$ & Neg & $\mathrm{Neg}$ & I & Neg & $=$ & Hemolysis \\
\hline 10 & $M$ & 58 & DN & Single & 558 & FK & $\mathrm{Neg}$ & Neg & $\mathrm{Neg}$ & $\mathrm{Neg}$ & Neg & $\uparrow$ & - \\
\hline 11 & $M$ & 52 & Mesangial GN & Single & 85 & CsA & $\mathrm{Neg}$ & $\mathrm{Neg}$ & $\mathrm{Neg}$ & $\mathrm{Neg}$ & $\mathrm{Neg}$ & $\uparrow$ & - \\
\hline 12 & $M$ & 36 & Focal GN & Single & na & FK & $\mathrm{Neg}$ & $\mathrm{Neg}$ & $\mathrm{Neg}$ & - & - & - & - \\
\hline 13 & $\mathrm{~F}$ & 40 & Focal GN & Single & 17 & FK & $\mathrm{Neg}$ & Neg & $\mathrm{Neg}$ & - & - & - & Hemolysis \\
\hline 14 & $\mathrm{~F}$ & 40 & ESRD & Single & 9 & FK & $\mathrm{Neg}$ & $\mathrm{Neg}$ & $\mathrm{Neg}$ & - & - & $\uparrow$ & Neg \\
\hline 15 & $M$ & 71 & Hypertensive & Single & 27 & FK & $\mathrm{Neg}$ & $\mathrm{Neg}$ & $\mathrm{Neg}$ & Neg & $\mathrm{Neg}$ & $\uparrow$ & Hemolysis \\
\hline 16 & $\mathrm{~F}$ & 62 & MPGN & Double & 336 & FK & $\mathrm{Neg}$ & $\mathrm{Neg}$ & $\mathrm{Neg}$ & $\mathrm{Neg}$ & $\mathrm{Neg}$ & $\uparrow$ & Hemolysis \\
\hline 17 & $\mathrm{~F}$ & 43 & GIN & Single & 626 & FK & $\mathrm{Neg}$ & $\mathrm{Neg}$ & Neg & II & $\mathrm{Neg}$ & $=$ & - \\
\hline ТОТ & Male & Mean \pm SD & & $S / D$ & Mean \pm SD & mTORi & Pos & Pos & Pos & DSAII & Pos & $\uparrow L D H$ & Hemolysis \\
\hline 17 & 9 & $51 \pm 12$ & & $15 / 2$ & $276 \pm 377$ & 8 & 0 & 0 & $0^{*}$ & 2 & 2 & 11 & 5 \\
\hline
\end{tabular}

Abbreviations: CMV, cytomegalovirus; CsA, cyclosporine; DSA, donor-specific antibody; ESRD, end-stage renal disease; EVE, everolimus; F, female; GIN, granulomatous interstitial nephritis; HIV, human immunodeficiency virus; HR, histology compatible with rejection; LDH, lactate dehydrogenase; M, male; MPGN, membranoproliferative glomerulonephritis; Neg, negative; Pos, positive; SLE, systemic lupus erythematosus; TMA, thrombic

$P<.05$ between drug-related and not drug-related groups. 
Table 2. Renal Graft Recipients With TMA That Is Not Drug Related

\begin{tabular}{|c|c|c|c|c|c|c|c|c|c|c|c|c|c|}
\hline Patient & Sex & Age $(y)$ & $\begin{array}{l}\text { Native Renal } \\
\text { Disease }\end{array}$ & Allograft & $\begin{array}{c}\text { TMA Onset } \\
\text { Posttransplant (d) }\end{array}$ & Prebiopsy Therapy & CMV & HIV & $\mathrm{HR}$ & DSA & C4d & LDH & Blood Smear \\
\hline 1 & $\mathrm{~F}$ & 59 & ESRD & Single & 19 & CsA & $\mathrm{Neg}$ & $\mathrm{Neg}$ & Pos & - & - & - & - \\
\hline 2 & $M$ & 39 & ESRD & Single & 187 & CsA & Neg & Neg & Pos & - & - & - & $\mathrm{Neg}$ \\
\hline 3 & $M$ & 51 & HIVAN & Single & 23 & CsA & $\mathrm{Neg}$ & Pos & $\mathrm{Neg}$ & Neg & $\mathrm{Neg}$ & - & Hemolysis \\
\hline 4 & $\mathrm{~F}$ & 56 & ESRD & Single & 10 & FK & $\mathrm{Neg}$ & $\mathrm{Neg}$ & Pos & $I+I I$ & Pos & $\uparrow$ & Hemolysis \\
\hline 5 & $M$ & 52 & ESRD & Single & 969 & CsA & $\mathrm{Neg}$ & $\mathrm{Neg}$ & Pos & - & - & - & Hemolysis \\
\hline 6 & $M$ & 34 & ESRD in $\mathrm{HCV}+$ & Single & 8 & CsA & $\mathrm{Neg}$ & $\mathrm{Neg}$ & Pos & I & Pos & - & Hemolysis \\
\hline 7 & $M$ & 62 & GN & Single & 2569 & CsA & $\mathrm{Neg}$ & $\mathrm{Neg}$ & Pos & I & Pos & $=$ & - \\
\hline 8 & $M$ & 32 & VUR & Single & 115 & FK & $\mathrm{Neg}$ & $\mathrm{Neg}$ & Pos & Neg & - & - & - \\
\hline 9 & $M$ & 77 & Hypertensive & Single & 2768 & CsA & $\mathrm{Neg}$ & $\mathrm{Neg}$ & Pos & - & - & $=$ & - \\
\hline 10 & $M$ & 40 & IgAN & Single & 2962 & CsA & Neg & $\mathrm{Neg}$ & $\mathrm{Neg}$ & $I+I \mid$ & Pos & $=$ & Hemolysis \\
\hline 11 & $\mathrm{M}$ & 54 & Extracapillary GN & Living & 2463 & FK & Neg & Neg & Pos & Neg & $\mathrm{Neg}$ & $=$ & - \\
\hline 12 & $\mathrm{M}$ & 60 & V-U reflux & Single & 11 & Thymogl. & Neg & Neg & Pos & - & - & - & - \\
\hline 13 & $\mathrm{M}$ & 48 & HIVAN & Single & 3 & EVE & Neg & Pos & $\mathrm{Neg}$ & Neg & $\mathrm{Neg}$ & $=$ & Hemolysis \\
\hline 14 & $\mathrm{M}$ & 71 & ESRD & Double & 15 & EVE & Pos & $\mathrm{Neg}$ & Neg & Neg & Neg & $=$ & Hemolysis \\
\hline 15 & $\mathrm{~F}$ & 52 & Atypical HUS & Single & 144 & Sir & $\mathrm{Neg}$ & Neg & Neg & - & Neg & - & - \\
\hline 16 & $M$ & 50 & Focal GN & Single & 199 & FK & Pos & Neg & Neg & - & - & - & $\mathrm{Neg}$ \\
\hline 17 & $M$ & 37 & SLE & Single & 3178 & CsA & $\mathrm{Neg}$ & Neg & Neg & I & Pos & $=$ & - \\
\hline 18 & M & 50 & DN, HIVAN & Single & 653 & $\mathrm{Cs} A+\mathrm{EVE}$ & Neg & Pos & Neg & Neg & Neg & - & - \\
\hline 19 & $M$ & 65 & Cryo GN & Single & 10 & MMF & Neg & $\mathrm{Neg}$ & Pos & Neg & Neg & $\uparrow$ & $\mathrm{Neg}$ \\
\hline Total & Male & Mean \pm SD & & S/D & Mean \pm SD & mTORi (EVE/Sir) & Pos & Pos & Pos & DSAII & Pos & $\uparrow \mathrm{LDH}^{*}$ & Hemolysis \\
\hline 19 & 16 & $52 \pm 12$ & & $18 / 1$ & $858 \pm 1217$ & $4(3 / 1)$ & 2 & 3 & $11^{\dagger}$ & 2 & 5 & 2 & 7 \\
\hline
\end{tabular}

Abbreviations: CMV, cytomegalovirus; CsA, cyclosporine; DSA, donor-specific antibody; ESRD, end-stage renal disease; EVE, everolimus; F, female; GIN, granulomatous interstitial nephritis; HIV, human immunodeficiency virus; HR, histology compatible with rejection; LDH, lactate dehydrogenase; M, male; MPGN, membranoproliferative glomerulonephritis; Neg, negative; Pos, positive; SLE, systemic lupus erythematosus; TMA, thrombotic microangiopathy; VUR, vesicoureteral reflux.

"Considered elevated if $>400 \mathrm{UI} / \mathrm{L}$

${ }^{\dagger} P<.05$ between drug-related and not drug-related groups. 
TMA and $10.7 \pm 2.1 \mathrm{ng} / \mathrm{mL}$ in patients without TMA (Fig 1). Receiver-operating characteristic analysis found a significant threshold of $12.5 \mathrm{ng} / \mathrm{mL}$ (area under the curve, $0.803 ; P=$ .006) (Fig 2). Tacrolimus (FK) TLC mean blood levels were $7.2 \pm 1.3 \mathrm{ng} / \mathrm{mL}$ in patients with TMA and $4.7 \pm 0.8 \mathrm{ng} / \mathrm{mL}$ in patients without TMA $(P=.0075)$. Similarly, EVE TLC in these patients was $15.3 \pm 8.1 \mathrm{ng} / \mathrm{mL}$ in patients with TMA and $4.9 \pm 0.9 \mathrm{ng} / \mathrm{mL}$ in patients without TMA $(P=.0011)$. $\mathrm{EVE} \mathrm{TLC} \mathrm{+} \mathrm{FK} \mathrm{TLC} \mathrm{was} 21.1 \pm 11.3 \mathrm{ng} / \mathrm{mL}$ in patients with TMA and $9.6 \pm 1.3 \mathrm{ng} / \mathrm{mL}$ in patients without TMA.

\section{DISCUSSION}

The goal of this study was to investigate the role of IS drugs in posttransplant TMA. We selected a TMA population not influenced by classical risk factors (eg, AMR, viral infections, vasculitides, systemic lupus erythematosus, recurrence of atypical HUS), in whom the role of the antirejection medication appeared predominant. However, we could not exclude the possible presence of underlying causes unknown at the time of onset.

According to the literature, drug-induced HUS represents $13 \%$ of all HUS cases [7]. The real incidence of drugrelated TMA in renal graft recipients is not known; renal biopsy is the only available method for diagnosis of TMA, and indications for renal biopsy vary among medical centers. The overall prevalence of TMA observed in our center since 1998 is $7.3 \%$, which is in line with the reported series in several studies [7-9].

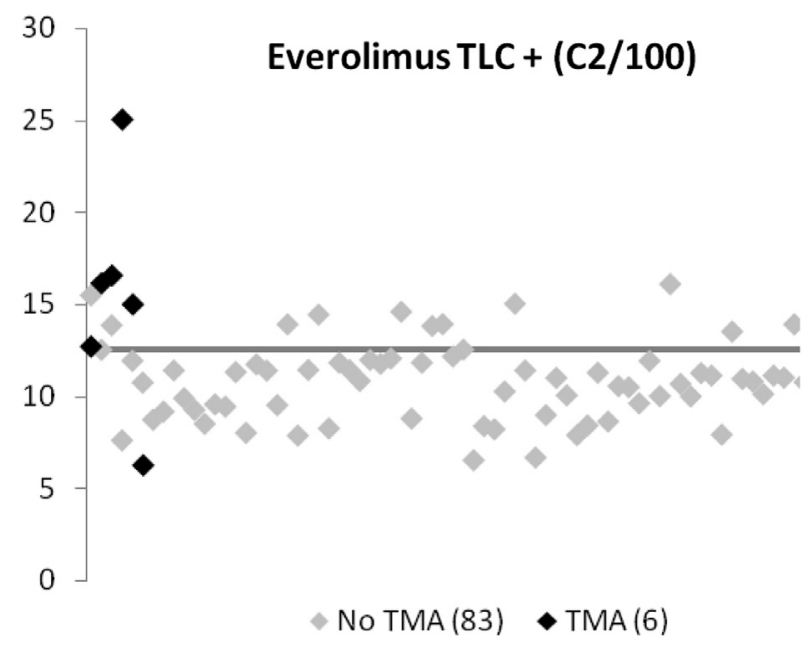

Fig 1. Summation of blood levels of everolimus TLC $+(C 2 / 100)$ in patients with drug-related thrombotic microangiopathy (TMA) treated with the combination of cyclosporine (CsA) and everolimus. Black diamonds represent $\Sigma$ blood levels in patients with TMA; grey diamonds represent $\Sigma$ blood levels in graft recipients without TMA. Vertical axis indicates blood concentration of drug expressed in nanograms per milliliter. The number of patients examined for each group is indicted in brackets. The line represents the $\Sigma$ safety threshold of $12.5 \mathrm{ng} / \mathrm{mL}$ [everolimus TLC + (C2/100)] suggested by the receiver-operating characteristic analysis.

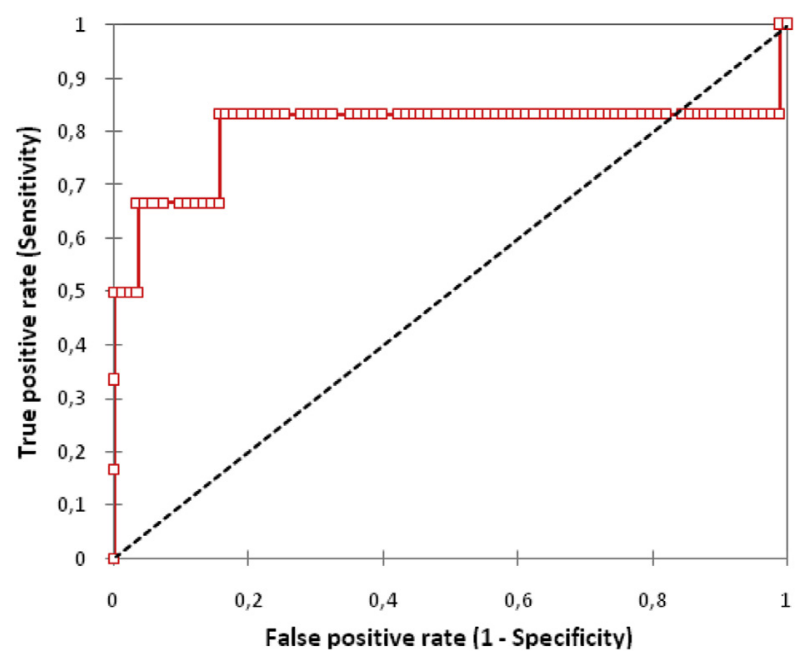

Fig 2. Receiver-operating characteristic curve of association of everolimus TLC $+(\mathrm{C} 2 / 100)\left(\sum\right)$ in patients exposed to mTOR inhibitors. Area under the curve, 0.803; $P=.006$.

The association between TMA and CsA is well established [10]. Although the prevalence of TMA in patients exposed to mTORi in our study was greater $(5.3 \%$ vs $2.8 \%$ in patients exposed to CNI only), these differences were not statistically significant. The data regarding exposure to IS could provide an explanation of this difference. Analyzing blood levels of drugs in relation to the presence of TMA, we found significantly higher drug blood levels in patients with TMA. Apart from the well-known relationship with CsA basal levels, the values of the $\Sigma$ are noteworthy; they reached an average of $15.2 \mathrm{ng} / \mathrm{mL}$ in patients with TMA, and in patients without TMA, the average summation was $10.7 \mathrm{ng} / \mathrm{mL}(P<.05)$.

Combined monitoring of IS drugs has recently proved to be a useful tool for reducing the risk of rejection within the first months after transplant [11]. The results of the present study support the use of combined monitoring to also predict and prevent the onset of de novo HUS. In fact, TMA in renal allograft recipients treated with mTORi is very infrequent below specific values $12.5 \mathrm{ng} / \mathrm{mL}$ of $\Sigma$ (Fig 1). Toxic effects of CNI and mTORi interact in a synergistic way. We hypothesize that the endothelial injury is due to the action of a constant high level of CsA amplified by EVE-mediated inhibition of endothelial cell regeneration. In other words, endothelial damage caused by CsA does not heal under the effect of mTORi. A similar interaction should also be assumed for the association of EVE-FK, but the low number of such cases in our study does not allow us to draw conclusions.

EVE-based IS therapy is an important prevention strategy of chronic drug-induced renal toxicity, allowing minimization of CNI doses. However, as well as SIR, EVE therapy seems to play a predisposition role in the onset of de novo TMA. A specific nephrotoxicity of mTORi has not been documented to be present on undamaged 
endothelium. As reported in the literature for SIR, EVE may be a causal factor in kidneys suffering from ischemic damage, such as double transplant, or marginal donors [5]. In this study, 2 cases fit this category: 1 double transplant and 1 extended-criteria donor.

Our study is limited by its retrospective design and the low number of cases. However, our results are strengthened by the use of a novel cognitive tool, represented by the combined monitoring of 2 drugs.

In conclusion, according to our data, TMA is a rare condition in the setting of renal transplant compared with the field of lung transplantation. Maintaining lower drug exposure could be the explanation for the different outcome. In fact, CsA TLC as reported by the German group (200-250 ng/mL) corresponds to $1000 \mathrm{ng} / \mathrm{mL}$ of C (concentration) $)_{\max }$ and therefore means no minimization of CNI. On the contrary, in our minimization protocol, CsA $\mathrm{C}_{\max }(\mathrm{C} 2)$ is maintained at $\sim 300$ to $350 \mathrm{ng} / \mathrm{mL}$. We also postulate a sequence of endothelial damage by $\mathrm{CNI}$, on which EVE fits hindering the repair of endothelial injury.

High blood levels of CNI and mTORi seem to predispose patients to posttransplant TMA. Combined monitoring of these 2 drugs might be useful for preventing this complication. Summated blood levels of [everolimus TLC $+(\mathrm{C} 2 /$ 100)] greater than $12.5 \mathrm{ng} / \mathrm{mL}$ should be avoided.

\section{REFERENCES}

[1] Ponticelli C, Banfi G. Thrombotic microangiopathy after kidney transplantation. Transplant Int 2006;19:789-94.

[2] Satoskar AA, Pelletier R, Adams P, Nadasdy GM, Brodsky S, Pesavento T, et al. De novo thrombotic microangiopathy in renal allograft biopsies-role of antibody-mediated rejection. Am J Transplant 2010;10:1804-11.

[3] McCauley J, Bronsther O, Fung J, Todo S, Starzl TE. Treatment of cyclosporin-induced haemolytic uraemic syndrome with FK506. Lancet 1989;2:1516.

[4] Keller K, Daniel C, Schöcklmann H, Endlich KH, Kerjaschki D, Johnson RJ, et al. Everolimus inhibits glomerular endothelial cell proliferation and VEGF, but not long-term recovery in experimental thrombotic microangiopathy. Nephrol Dial Transplant 2006;21:2724-35.

[5] Sartelet H, TO. Sirolimus-induced thrombotic microangiopathy is associated with decreased expression of vascular endothelial growth factor in kidneys. Am J Transplant 2005;5: 2441-7.

[6] Lovric S, Kielstein JT, Kayser D, Bröcker V, Becker JU, Hiss M, et al. Combination of everolimus with calcineurin inhibitor medication resulted in post-transplant haemolytic uraemic syndrome in lung transplant recipients-a case series. Nephrol Dial Transplant 2011;26:3032-8.

[7] Terrell DR, Williams LA, Vesely SK, et al. The incidence of thrombotic thrombocytopenic purpura-hemolytic uremic syndrome: all patients, idiopathic patients, and patients with severe ADAMTS13 deficiency. J Thromb Haemost 2005;3:1432-6.

[8] Hachem RR, Yusen RD, Chakinala MM, et al. Thrombotic microangiopathy after lung transplantation. Transplantation 2006;81:57-63.

[9] Young BA, Marsh CL, Alpers CE, Davis CL. Cyclosporineassociated thrombotic microangiopathy/hemolytic uremic syndrome following kidney and kidney-pancreas transplantation. Am J Kidney Dis 1996;28:561-71.

[10] Noel C, Sauneir P, Hazzan M, Pruvot F, Dracon M, Jude B, Lelievre G. Incidence and clinical profile of microvascular complications in renal allograft patients treated with cyclosporine. Ann Med Interne (Paris) 1992;143:33-6.

[11] Corbetta et al. Exposure to everolimus and not to cyclosporine is associated with freedom from acute rejection in de novo renal recipients. ATC Toronto 2008; ICT Sidney 2008. Corbetta et al 2007 AST. 